\title{
Longer term quality of life and outcome in stroke patients: is the Barthel index alone an adequate measure of outcome?
}

Peter R Wilkinson, Charles D A Wolfe, Fiona G Warburton, Anthony G Rudd, Robin S Howard, Ralph W Ross-Russell, Roger Beech

\begin{abstract}
Objectives-To consider whether the Barthel Index alone provides sufficient information about the long term outcome of stroke.
\end{abstract}

Design-Cross sectional follow up study with a structured interview questionnaire and measures of impairment, disability, handicap, and general health. The scales used were the hospital anxiety and depression scale, mini mental state examination, Barthel index, modified Rankin scale, London handicap scale, Frenchay activities index, SF36, Nottingham health profile, life satisfaction index, and the caregiver strain index.

Setting-South east London.

Subjects-People, and their identified carers, resident in south east London in 1989-90 when they had their first in a lifetime stroke aged under 75 years.

Interventions-Observational study.

Main outcome measures-Comparison and correlation of the individual Barthel index scores with the scores on other outcome measures.

Results-One hundred and twenty three $(42 \%)$ people were known to be alive, of whom $106(86 \%)$ were interviewed. The median age was 71 years (range 34-79). The mean interval between the stroke and follow up was 4.9 years. The rank correlation coefficients between the Barthel and the different dimensions of the SF36 ranged from $r=0.217$ (with the role emotional dimension) to $r=0.810$ (with the physical functioning dimension); with the Nottingham health profile the range was $r=-0.189$ (with the sleep dimension, NS) to $r=-0.840$ (with the physical mobility dimension); with the hospital and anxiety scale depression component the coefficient was $r=-0.563$, with the life satisfaction index $r=0.361$, with the London handicap scale $r=0.726$ and with the Frenchay activities index $r=0.826$.

Conclusions-The place of the Barthel index as the standard outcome measure for populations of stroke patients is still justified for long term follow up, and may be a proxy for different outcome measures intended for the assessment of other domains.

(Quality in Health Care 1997;6:125-130)

Keywords: stroke; outcome; quality of life

\section{Introduction}

Recently there has been increasing interest in the measurement of outcomes of health care. This is as true for stroke medicine as it is for any other specialty. The Key Area Handbook for coronary heart disease and stroke produced by the Department of Health ${ }^{1}$ suggested action to streamline the assessment of people with strokes. These were the development of "common assessment procedures throughout the rehabilitation process" and for "a standard assessment procedure which could act as the basis of outcome measures, against which performance could be monitored". The implication of these statements is that providers of services to stroke patients, be they health carers, social services, or others, should work together to develop uniformly agreed policies and outcome assessments.

Quality of life is difficult to define but de Haan et al ${ }^{2}$ suggest that over time a broad consensus has arisen as to which dimensions should be included in assessing the quality of life of stroke patients; physical, functional, psychological, and social health. The available measures are not always specifically aimed at one of these dimensions alone. An alternative framework for measurements of stroke outcome is the classification of impairments, disabilities, and handicaps of the World Health Organisation (WHO). ${ }^{3}$

The objective of the present study was to consider whether the Barthel index alone provides sufficient information about the long term outcome of stroke. This should help decide which outcome measures are the most pragmatic and appropriate for assessing the long term outcome of stroke patients.

\section{CHOICE OF OUTCOME MEASURES USED IN THIS} STUDY

Wade $^{4}$ suggested that the ideal stroke outcome measure should be simple to administer, valid and reliable, sensitive to clinically relevant change, relevant to both user and patient, and understood by a non-specialist. Very few of the available scales satisfy these requirements.

The Barthel index ${ }^{5}$ is a leading contender as an outcome measure for populations of stroke patients, and most closely satisfies these criteria. ${ }^{4}$ It is valid and reliable, simple to administer, and easily understood and consequently has become an accepted outcome measure in stroke research. The scale assesses 10 activities of self care and daily activity. The maximum score of 20 suggests that the subject 
is functionally independent. The functional basis of the Barthel index allows an estimate of the level of disability and gives some insight into the likely need for services. However, the Barthel index lacks any assessment of the ability to carry out tasks in the community or any measures of communication, mental and emotional factors, or of family involvement.

The mini mental state examination ${ }^{6}$ assesses cognitive function. It has been widely used and validated. The maximum score is 30 . People with a normal mental state have high scores. The hospital anxiety and depression scale ${ }^{7}$ is a valid measure of anxiety and depression. Scores between 8 and 10 were considered borderline and scores of 11 or more as indicating depression or anxiety.

The Frenchay activities index was included as a measure of social function and of handicap. ${ }^{8}$ It was designed specifically for stroke patients. Although there was no prestroke assessment to allow for changes over time the index provided a framework of activities to ask the patients about. A scoring system with a minimum score of 15 and a maximum of 60 was used. The higher the score the greater the number of activities done. The modified Rankin scale ${ }^{9}$ is used as a handicap measure. It has many limitations and combines impairment, disability, and dependency into one score. Scores range from 0 to 5 . A score of 0 to 2 indicates functional independence and a score of 5 indicates severe handicap. The London handicap scale is a recently developed scale $^{10}$ which uses a questionnaire to assess six different dimensions of handicap. Each dimension has its own scale but a formula with scale weights calculates an overall handicap score of between 0 (maximum handicap) and 1 (minimum). Initial assessments of validity and reliability with stroke patients have been satisfactory. ${ }^{11}$ The scale is intended for group rather than individual assessment.

There are many different measures of self perceived health. The short form 36 (SF36) allows the domains of physical and social functioning and mental health to be assessed as well as the effects of physical and mental health on the daily activities of stroke patients. ${ }^{12}$ Each dimension is scored from 0 to 100 . A high score suggests good health. Assessment of the validity and reliability of the SF36 is ongoing, but satisfactory to date.

Suggestions have been made about how to improve the SF36 for use with the elderly people. ${ }^{13}$ Both the original and modified versions of the SF36 were used in this study. The Nottingham health profile (NHP) ${ }^{14}$ has been used with stroke patients. ${ }^{15}$ Only the first part of the Nottingham health profile was used in this study as there are reservations about the reliability of the second part. ${ }^{16}$ The Nottingham health profile assessments include the domains of energy, emotion, social isolation, and physical mobility. Each dimension is scored between 0 and 100. A high score suggests poor health. The life satisfaction index ${ }^{17}$ was included as an example of a single global self assessment of outcome. A total overall score for life satisfaction is calculated from eight questions. Four of the questions are interpreted as measuring "acceptancecontentment" and the remainder as "achievement-contentment".

For carers the first part of the Nottingham health profile and the hospital anxiety and depression scale were used. The caregiver strain index ${ }^{18}$ requires further work on its validity and reliability, but was included as it provides information not just about strain on carers but also some of the particular problems carers face. This could help identify the support and services carers require.

A wide range of scales have been selected for investigation. Some such as the hospital anxiety and depression scale, and Barthel and Frenchay activities index aim to assess impairment, disability, and handicap respectively. Others such as the Nottingham health profile and SF36 cannot be classified so easily. Not all the domains of all the scales can be translated into service provision for stroke patients. Purchasers will be particularly interested in those instruments which are valid, reliable, acceptable both in terms of cost and ease of completion, and are easily interpreted as outcomes or health needs in terms of service provision. Table 1 shows the final choice of scales used in this study.

As well as the scales two simple stroke questions were included in the interview questionnaire. ${ }^{19}$ The questions were designed to be used in the follow up of large groups of stroke patients. The questions were "in the last two weeks did you require help from another person for everyday activities?" and "do you feel that you have made a complete recovery from your stroke?".

ESTABLISHING THE COMMUNITY BASED STROKE REGISTER

In 1989-90 a community based stroke register was established in south east England ${ }^{20}$ which provided a cohort for this follow up study in $1994 / 1995$. The methodology used to establish the register in 1989 has been previously described. ${ }^{20}$ The World Health Organisation (WHO) definition of stroke was used. ${ }^{21}$ People who had had their first ever stroke aged under 75 years were entered including those who did not survive the initial event. Cases were notified by general practitioners, district nurses, the rehabilitation services, and hospital consultants. Field workers reviewed accident and emergency registers and visited hospital wards. Hospital and coroners' postmortem reports and the local district death certificates were also reviewed. Information about the patients was collected about their demography, their premorbid level of disability and handicap, their maximum clinical impairment, and the therapy services they received. All the patients were reviewed at the time of their stroke and after three months.

\section{Methods}

ASSESSMENTS

In the present study only those survivors who had lived in London at the time of their stroke were traced and interviewed. Subjects who had 
Table 1 Outcome scales used in the study

\begin{tabular}{|c|c|c|}
\hline Scale & What it measures & Reason for inclusion \\
\hline SF36 & General health & Broad outcome measure \\
\hline Nottingham health profile & General health & $\begin{array}{l}\text { Broad measure. Used with } \\
\text { stroke patients }\end{array}$ \\
\hline Life satisfaction index & Quality of life & Single global measure \\
\hline $\begin{array}{l}\text { Hospital anxiety and depression } \\
\text { scale }\end{array}$ & Psychological impairment & $\begin{array}{l}\text { Assesses both depression and } \\
\text { anxiety }\end{array}$ \\
\hline Mini mental state examination & Cognitive impairment & Standard measure \\
\hline Barthel index & Disability & $\begin{array}{l}\text { Standard stroke outcome } \\
\text { measure }\end{array}$ \\
\hline Modified Rankin scale & Handicap & Widely used in stroke studies \\
\hline Frenchay activities index & Handicap & $\begin{array}{l}\text { Stroke specific measure } \\
\text { Details some aspects of } \\
\text { community involvement }\end{array}$ \\
\hline London handicap scale & Handicap & A new scale \\
\hline Caregiver strain index & General health & $\begin{array}{l}\text { Carer specific scale. Indicates } \\
\text { some causes of strain and stress }\end{array}$ \\
\hline
\end{tabular}

moved from London, but who lived anywhere in the United Kingdom, were included. The subjects completed a structured interview questionnaire and the various scales were assessed face to face at the same interview. All the interviews were completed in 1994- 5 by one investigator (PW). The scales were completed in the same order, and most were read out to the subjects. The original and modified versions of the SF36 were allocated randomly with a block technique. The project received local ethical approval and the subjects gave their informed consent.

ANALYSIS OF THE DATA

The data were analysed primarily at the individual level, but some analyses were based on groups of subjects categorised according to the disability categories based on the Barthel index as suggested by Wade and Langton-Hewer. ${ }^{22}$ The nature of the scales used meant that the subjects' scores were not normally distributed and therefore nonparametric tests were used for most of the analyses.

\section{PSYCHOLOGICAL IMPAIRMENT}

The subjects' scores on the mini mental state examination were described by disability category. The correlation between the subjects' individual scores on the hospital anxiety and depression scale with their Barthel score were explored with the Spearman rank correlation coefficient. The $\chi^{2}$ test was used to assess the association of disability with anxiety and depression.

\section{HANDICAP}

Rank correlation was used to assess the correlation between the individual subjects' scores on the London handicap scale and the Frenchay activities index with their Barthel index scores. The same test was used to assess the correlation between the modified Rankin scale and the London handicap scale.

SF36 AND THE NOTTINGHAM HEALTH PROFILE The differences between the two versions of the SF36 were compared with the Mann-Whitney $U$ test. Rank correlation was used to assess the correlation between the subjects' individual scores on the various dimensions of the SF36 and Nottingham health profile with their indi- vidual Barthel scores. The Wilcoxon matched pairs test was used to compare the scores on the SF36 and Nottingham health profile with the predicted norms.

\section{LIFE SATISFACTION INDEX}

Rank correlation was used to assess the correlation between the individual person's scores and their Barthel scores.

\section{TWO SIMPLE STROKE QUESTIONS}

The sensitivity and specificity of the two questions were assessed with a modified Rankin score of 3-5 as suggesting help would be required, and a score of $0-2$ as indicating that it would not. A modified Rankin score of 0 was taken as indicating a complete recovery. For the "any help" question a Barthel score of 0-19 was taken as indicating the need for help with 20 indicating functional independence.

\section{CARERS}

The results from the carers were analysed with the same tests as the subjects.

\section{Results}

Of the 291 people aged under 75 years originally registered in 1989-90, 154 (53\%) had died and $14(5 \%)$ were lost to follow up. Of the remaining $123,106(86 \%)$ were interviewed in person (the remaining 17 either declined or their general practitioner did so on their behalf). In six cases the informant was either the main carer or a healthcare professional and the subjective quality of life scales were not completed.

The median age of the people interviewed at the time of their stroke was 66 years (range 28-74), and at follow up 71 years (range 34-79). The mean interval between the stroke and the assessments was 4.9 years with a range of 4.3 to 5.5 years. Fifty seven (54\%) subjects were male, $89(84 \%)$ were white, and $13(12 \%)$ West Indian or African. Fifty six (53\%) subjects described themselves as married, and $77(73 \%)$ as retired. Only five (5\%) people were in full time employment. Fifty (47\%) subjects identified a main carer, usually a spouse $(72 \%)$, son, or daughter $(20 \%)$. Twenty nine carers $(58 \%)$ were women. The median age of the carers was 65 years with a range of 26 to 87 .

With the disability categories based on the Barthel index score 36 (34\%) people were functionally independent (a score of 20), 39 (37\%) mildly disabled (a score of 15-19), 17 (16\%) moderately disabled (a score of 10-14), and $14(13 \%)$ severely disabled (a score of 0-9). Information was available about the Barthel score at three months after their stroke for 103 subjects. Fifty six (54\%) subjects were in the same disability category at five years as they were at three months, but $40(39 \%)$ had deteriorated by at least one category. The remaining seven $(7 \%)$ had improved.

Twelve people did not complete all the quality of life scales, either because they were physically unable to complete the forms and unable to make their responses understood, or the interview was ended at their request. Six 
were severely disabled, three moderately disabled, and two mildly disabled. The remaining person was functionally independent and completed all except one question on the London handicap scale. Those people who did not complete the scales were generally more disabled than those who did. In interpreting the following results it should be remembered that the distribution, if those who did not complete the questionnaires had been included, would probably have moved towards a poorer quality of life.

\section{PSYCHOLOGICAL IMPAIRMENT}

Ninety eight $(92 \%)$ people completed the mini mental state examination. Of these $27(28 \%)$ scored $\leqslant 24$, suggesting a possible cognitive disorder. ${ }^{23}$ The importance of this finding is complicated by some of the subjects being unable to complete some sections because of arm weakness. Rather than exclude over a quarter of the subjects it was decided to include those who scored $\leqslant 24$ in the overall analysis. The median scores and ranges for the mini mental state examination for the disability categories based on the Barthel index were severe 23 (14-29), moderate 26 (6-29), mild 28 (1930), and functionally independent 28 (20 30).

The hospital anxiety and depression scale was completed by 96 (91\%) subjects. The number of people identified as being anxious or depressed for each disability category was small, but only six of the 36 functionally independent subjects had scores in the depressed or borderline depressed categories compared with 29 of the 60 with some disability $\left(\chi^{2}=9.739\right.$, $\mathrm{df}=1, \mathrm{P}=0.0018$ ). For the anxiety scale the numbers were seven and 23 respectively $\left(\chi^{2}=3.737, \mathrm{df}=1, \mathrm{P}=0.0532\right)$. The correlation coefficient between the subjects' Barthel score and their hospital anxiety and depression scale score was much stronger for the depression scale $(r=-0.563 \mathrm{p}<0.001 \mathrm{df}=94)$ than for the anxiety scale $(r=-0.187, \mathrm{P}>0.05, \mathrm{df}=94)$.

\section{HANDICAP}

With the modified Rankin scale 38 (36\%) people were functionally independent (scores of $0-2$ ). The London handicap scale scores were correlated with the individual subjects' modified Rankin category $(r=0.784, \mathrm{P}<0.001, \mathrm{df}=$ 94) and with their Barthel scores $(r=0.726$, $\mathrm{P}<0.001$, df =94).

Most people with a moderate or more severe disability carried out few of the activities in the Frenchay activities index. Fourteen (13\%) people scored 15, 37 (35\%) 16-30, 39 (37\%) $31-45$, and $16(15 \%) 46-55$. The correlation coefficient between the individual scores on the Frenchay activities index and the Barthel index was $0.826(\mathrm{P}<0.001, \mathrm{df}=104)$.

\section{SHORT FORM 36 AND THE NOTTINGHAM HEALTH} PROFILE

The SF36 was completed by 97 subjects. Although the scores tended to be higher for the modified version there was no significant difference between the two scores on any of the dimensions and for the analysis the results have been combined. All the various dimensions of the SF36 showed a significant correlation between the individual subject's scores and their Barthel score (table 2). The Nottingham health profile was completed by 96 respondents. All the dimensions, apart from that for sleep, had a significant correlation with the Barthel score (table 2).

The only significant correlations between the various dimensions and the patient's age at interview were with "role-emotional" on the SF36 $(P<0.02)$, and with sleep $(P<0.05)$, on the Nottingham health profile.

Tables 3 and 4 give the median difference between the scores of the subjects on the dimensions of the SF36 and Nottingham health profile and the expected scores from age and sex matched interviewed normal values for the SF $36^{24}$ and from age, sex, and social class matched norms for the Nottingham health profile. $^{25}$

\section{LIFE SATISFACTION INDEX}

The life satisfaction index was completed by 95 subjects. The results suggest a trend of increasing overall satisfaction, and for both subscales, with decreasing disability. The correlation coefficient between the individual Barthel scores and the scores on the life satisfaction index were 0.361 for the overall total $(P<0.001$, df 93), $0.357 \quad(P<0.001)$ for the acceptance total, and $0.307(P<0.01)$ for the achievement total. The life satisfaction index was originally intended for elderly subjects, and similar results were obtained for patients aged 65 and over.

\section{TWO SIMPLE STROKE QUESTIONS}

These were completed for all 106 subjects. The sensitivity of the "any help in the last two weeks" question was 74\% (50/68) (95\% CI 63 to 84$)$ and the specificity $87 \%$ (33/38) (95\% CI 76 to 98 ) against the modified Rankin scale and $74 \%(52 / 70)(95 \% \mathrm{CI} 64$ to 85$)$ and $92 \%$ (33/36) (95\% CI 83 to 100$)$ respectively against the Barthel index. For the "complete recovery question" everyone with a modified Rankin score of 0 answered yes (9/9). The specificity was $75 \%$ (73/97) (95\% CI 67 to 84 ).

Table 2 The rank correlation coefficients between the individual Barthel index score at five years and the corresponding scores on the various dimensions of the SF36 and Nottingham health profile

\begin{tabular}{lll}
\hline & \multicolumn{2}{l}{$\begin{array}{l}\text { Rank correlation } \\
\text { coefficient } \\
\text { index }\end{array}$} \\
\cline { 2 - 3 } Qualith Barthel \\
\cline { 2 - 3 } & $r$ & Pvalue \\
\hline SF36 (n=97): & & \\
Physical functioning & 0.810 & $<0.001$ \\
Social functioning & 0.481 & $<0.001$ \\
Role: physical & 0.415 & $<0.001$ \\
Role: emotional & 0.217 & $<0.05$ \\
Mental health & 0.332 & $<0.01$ \\
Vitality & 0.500 & $<0.001$ \\
Bodily pain & 0.356 & $<0.001$ \\
General health & 0.438 & $<0.001$ \\
Nottingham health profile (n=96): & & \\
$\quad$ Energy & -0.605 & $<0.001$ \\
Pain & -0.499 & $<0.001$ \\
Emotion & -0.423 & $<0.001$ \\
Sleep & -0.189 & $>0.05$ \\
Social interaction & -0.460 & $<0.001$ \\
Physical mobility & -0.840 & $<0.001$ \\
\hline
\end{tabular}


Table 3 Median differences for SF36 scores for patients with stroke compared with normal values matched for age and sex

\begin{tabular}{lll}
\hline & Median difference (range) & P value \\
\hline Physical functioning & $-28.80(-91.6$ to +46.2$)$ & $<0.0001$ \\
Social functioning & $-20.80(-87.5$ to +29.2$)$ & $<0.0001$ \\
Mental health & $-11.60(-57.9$ to +26.4$)$ & $<0.0001$ \\
Vitality & $-11.10(-68.9$ to +52.4$)$ & 0.001 \\
Pain & $-9.30(-64.1$ to +39.7$)$ & 0.23 \\
General health & $0.00(-70.2$ to +53.4$)$ & 0.35 \\
Role physical & $-27.00(-87.1$ to +52.6$)$ & $<0.0001$ \\
Role emotional & $-44.20(-92.9$ to +22.5$)$ & $<0.0001$ \\
\hline
\end{tabular}

A negative value indicates that patients have worse health.

Table 4 Median differences for Nottingham health profile scores for patients with stroke compared with normal values matched for age, sex, and social class

\begin{tabular}{lll}
\hline & Median difference (range) & P value \\
\hline Energy & $23.30(-35.3$ to +93.4$)$ & $<0.0001$ \\
Pain & $-2.00(-19.0$ to +90.1$)$ & 0.041 \\
Emotion & $9.00(-15.7$ to +89.5$)$ & $<0.0001$ \\
Sleep & $0.95(-27.3$ to +85.9$)$ & 0.002 \\
Social isolation & $14.80(-13.4$ to +95.3$)$ & $<0.0001$ \\
Physical mobility & $31.24(-22.1$ to +88.9$)$ & $<0.0001$ \\
\hline
\end{tabular}

A positive value indicates that patients have worse health.

CARERS

Of the 50 carers, 36 (72\%) completed the Nottingham health profile, $37(74 \%)$ the hospital anxiety and depression scale, and 38 (76\%) the caregiver strain index. Of the 12 who did not complete any of the scales, one was caring for a patient with a Barthel score of 0-9, three for patients with a score of 10-14, and eight for patients with a score of 15-19.

There was no significant correlation between the carers' scores on any of the Nottingham health profile dimensions and the patients' Barthel scores. With the Wilcoxon matched pairs test the only significant difference between the carers' scores on the Nottingham health profile and the age and sex matched norms was for the emotion scale where the median difference was 6.22 (range -16.6 to +89.4, $\mathrm{P}=0.017$ ).

Twelve $(32 \%)$ of the 37 carers who completed the hospital anxiety and depression scale had borderline or clinically anxious scores. Six (16\%) had borderline or depressed scores. On the caregiver strain index eight (21\%) carers had scores of seven or more, suggesting that they were stressed.

\section{Discussion}

This study has described the long term quality of life of a community based population of stroke patients. There are no agreed definitions or scales for measuring quality of life. In an attempt to describe it with one or more scales, it is acknowledged that the subjects may feel the scales do not accurately reflect their own quality of life in terms of their individual circumstances and requirements.

Also, the study has considered whether it is justifiable to use the Barthel index as the only outcome measure because of its correlation with measures focusing on other domains. In assessing this through correlations it is acknowledged that those measures with dimensions or components which are strongly based on physical functioning are likely to have the strongest correlation. It should also be remembered that a significant correlation coefficient does not necessarily mean a very strong linear relation.

From a clinical viewpoint the measures of interest in the long term outcome of stroke have focused mainly on survival, recurrence, physical recovery, and the occurrence of complications. But interest in more general outcomes, such as quality of life, is being encouraged. The drawback of general measures, such as the SF36 and Nottingham health profile, is that it may be more difficult to equate a particular finding with either the structure or process involved in the care. A search of the medical literature for quality of life scales shows many possible instruments. In this area of work investigators tend to devise new measures rather than use those already available. Many of the instruments have not been adequately assessed for validity and reliability.

In 1987, a review of outcome measures in stroke rehabilitation research criticised the lack of use of broader measures. The authors drew attention to the limitations of assessing only physical recovery and self care and suggested that subjective measures of health status and quality of life should be included in assessments. ${ }^{26}$ Increasing a patient's quality of life is an important target of rehabilitation. Ebrahim $^{27}$ suggested that this may be equated with reducing handicap and that assessments of quality of life and of handicap may be more relevant than changes in impairment or disability.

This study considered the long term outcome of stroke in terms of the relation between measures of quality of life, impairment, and handicap, with disability as measured by the Barthel index. Overall the subjects' scores on the various scales were correlated with their disability. The major component of this disability is likely to have been related to the original stroke. The strongest correlation coefficients were between the Barthel index scores and the hospital anxiety and depression scale depression score, Frenchay activities index total, physical functioning and vitality dimensions on the SF36, physical mobility and energy dimensions on the Nottingham health profile, and the London handicap scale.

The weakest correlation coefficients were between the Barthel index scores and the "role-emotional" and "mental health" dimensions on the SF36 and the "sleep" and "emotion" dimensions on the Nottingham health profile. The correlation coefficients between the Barthel and the anxiety component of the hospital anxiety and depression scale as well as the life satisfaction index were also poor.

Not surprisingly the strongest correlations were between the Barthel index and those outcome measures most closely related to physical disability. The weaker correlations with the mental health and emotion components of the SF36 and Nottingham health profile reflect that the Barthel indexdoes not measure any psychological components. Despite this a reasonable correlation with the depression 
scale of the hospital anxiety and depression scale was found.

Therefore to achieve a truly comprehensive assessment of the long term outcome of stroke it is likely that outcome measures aimed at overall wellbeing and mental health would be required as well as the Barthel index. This will increase the burden on the patients and require additional resources. How easily the extra information obtained could be used to change provision of services is questionable.

What do these findings mean in terms of outcome measures for stroke patients? It seems that the place of the Barthel index as the standard outcome measure is still justified for long term follow up. This is particularly relevant for the provision of services. The Barthel index also has the advantages of being simple and quick to complete. However, the most suitable measures for research studies will depend on the question being asked. The additional information from further outcome measures is limited by their correlation with the Barthel scores. This could be interpreted as suggesting that a broad general measure, or handicap measure, could be used on its own as it would reflect the underlying disability. However, the disadvantage of the broader outcome measure is that it is not possible to relate scores to the process of care either to identify potential problems or to estimate the need for particular services. This is not as true of the Barthel index, which could also be used to estimate some of the service need - for example, for help with bathing, feeding, and toileting. A reasonable aim for rehabilitation is to help the patient return to their premorbid lifestyle. For this to be used as an outcome measure requires an assessment of the patient's premorbid state. This can be achieved with the Barthel index and the Frenchay activities index, but might not be possible for the general health measures.

As a result of the need to follow up many patients in a trial, Lindley $e t a l^{19}$ investigated by phone or by post the validity of two simple questions related to stroke, and concluded that such simple questions could be used to assess stroke outcomes. In this study the two simple stroke questions had comparable sensitivity and specificity to the original work for the help question, but a worse specificity for the recovery question. The confidence intervals for the various values do overlap. These differences may be due to the questions being asked in person and not by phone or post as in the study by Lindley et al. ${ }^{19}$ Further work is clearly needed but these findings are promising and may lead on to the use of reliable and valid simple patient self assessed outcome measures. The study by Lindley et $a l^{19}$ also used a postal and telephone version of the Barthel index, but the sensitivities of the questions were poor when judged against the assessment by the visiting nurse. Despite the potential problems of developing a valid and reliable telephone or postal version, the use of the Barthel index as the standard outcome measure may give some insight into the population's quality of life as well as their disability.

We thank all the subjects and their carers for their help with the study. We also thank local healthcare professionals for their help in establishing the original register and for their help in in establishing the original register and for their help in approaching the subjects. This study was funded by Research
and Development, Northern and Yorkshire Regional Health and Development, Northern and Yorks

1 Department of Health. Health of the Nation key area handbook: coronary heart disease and stroke. London: Department of Health, 1993.

2 de Haan R, Aaronson N, Limburg M, Langton-Hewer R, Crevel H. Measuring quality of life in stroke. Stroke 1993, 24:320-7.

3 World Health Organisation. The international classification of impairments, disabilities and handicaps. Geneva: WHO, 1980 .

4 Wade DT. Assessing disability after acute stroke. In: F Clifford Rose, ed. Stroke: epidemiological, therapeutic and socio-economic aspects. London: Royal Society of Medicine, 1986:101-14.

5 Mahoney F, Barthel D. Functional evaluation: the Barthel index. Md Med $\mathcal{f} 1965 ; 14: 61-5$.

6 Folstein M, Folstein S, McHugh P. "Mini-mental state": a practical method for grading the cognitive state of patients for the clinician. $\mathcal{F}$ Psychiatr Res $1975 ; 12: 189-98$.

7 Zigmond A, Snaith R. The hospital anxiety and depression scale. Acta Psychiatr Scand 1983;67:361-70.

8 Holbrook M, Skilbeck C. An activities index for use with stroke patients. Age Ageing 1983;12:166-70.

9 Bamford J, Sandercock P, Warlow C, Slattery J. Interobserver agreement for the assessment of handicap in stroke server agreement for the asse
patients. Stroke 1989;20:828.

10 Harwood R, Rogers A, Dickinson E, Ebrahim S. Measuring handicap: the London handicap scale, a new outcome measure for chronic disease. Quality in Health Care 1994;3 11-5.

11 Harwood R, Gompertz P, Ebrahim S. Handicap one year after a stroke: validity of a new scale. $\mathcal{F}$ Neurol Neurosur Psychiatry 1994;57:825-9.

12 Brazier J, Harper R, Jones N. O'Cathain A, Thomas K, Usherwood T, et al. Validating the SF36 health survey questionnaire: new outcome measure for primary care. BMF 1992;305:160-4.

13 Hayes V, Morris J, Wolfe C, Morgan M. The SF36 health survey questionnaire: is it suitable for use with older adults? survey questionnaire: is it suita

14 Hunt S, McKenna S, McEwen J, Backett E, Williams J, Papp E. A quantitative approach to perceived health status a validation study. $\mathcal{F}$ Epidemiol Community Health 1980;34: 281-6.

15 Ebrahim S, Barer D, Nouri F. Use of the Nottingham health profile with patients after stroke. $\mathcal{F}$ Epidemiol Community Health 1986;40:166-9.

16 Bowling A. Measuring health. Buckingham: Open University Press, 1991;4:56-92.

17 Bigot A. The relevance of American life satisfaction indices for research on British subjects before and after retirement. Age Ageing 1974;3:113-21.

18 Robinson B. Validation of a caregiver strain index. F Gerontol 1983;38:344-8.

19 Lindley R, Waddell F, Livingstone M, Sandercock P, Dennis $\mathrm{M}$, Slattery $\mathrm{J}$, et al. Can simple questions assess outcome after stroke? Cerebrovasc Dis 1994;4:314-24.

20 Wolfe C, Taub N, Woodrow J, Richardson E, Warburton F Burney P. Does the incidence, severity, or case fatality of stroke vary in southern England? $\mathcal{f}$ Epidemiol Communit Health 1993;47:139-43.

21 Hatano S. Experience from a multicentre stroke register: preliminary report. Bull World Health Organ 1976;54:541 53.

22 Wade D, Langton-Hewer. Functional abilities after stroke: measurement, natural history and prognosis. $\mathcal{F} \mathrm{Neurol} \mathrm{Neu}$ measurement, natural history and
rosurg Psychiatry 1987;50:177-82.

23 De Paulo J, Folstein M, Gordon B. Psychiatric screening on a neurological ward. Psychol Med 1980;10:125-32.

24 Lyons R, Fielder H, Littlepage B. Measuring health status with the SF36: the need for regional norms. F Public Health Med 1995;17:46-50.

25 The Nottingham health profile user's manual. Manchester: Galen Research, 1994

26 Seale C, Davies P. Outcome measurement in stroke rehabilitation research. International Disability Studies 1987 9:155-60.

27 Ebrahim S. Clinical epidemiology of stroke. Oxford: Oxford University Press, 1990. 